\title{
Recognizing and Managing Obstructive Sleep Apnea in Primary Care
}

\author{
Larry Culpepper, MD, MPH, and Thomas Roth, PhD
}

\begin{abstract}
Objective: This review aims to impart information regarding recognition of obstructive sleep apnea (OSA) and associated excessive sleepiness (ES) in the primary care setting in order to provide optimal care to patients with this common but serious condition. This review will also discuss the prevalence and treatment of depression in patients with OSA.
\end{abstract}

Data Sources: A MEDLINE search of articles published between 1990 and 2008 was conducted using the search terms obstructive sleep apnea AND excessive sleepiness, obstructive sleep apnea AND depression, and obstructive sleep apnea AND primary care. Searches were limited to articles in English concerned with adult patients.

Study Selection: In total, 239 articles were identified. Articles concerning other sleep disorders and forms of apnea were excluded. The reference lists of identified articles were searched manually to find additional articles of interest.

Data Synthesis: Primary care physicians can aid in the diagnosis of OSA and associated ES by being vigilant for lifestyle and physical risk factors associated with this condition. In addition, primary care physicians should maintain a high level of clinical suspicion when presented with illnesses that are commonly comorbid with OSA, such as psychiatric disorders and depression, in particular. Conversely, assessment of patients with OSA for common comorbidities may also improve a patient's prognosis and quality of life.

Conclusions: Primary care physicians play a vital role in recognizing OSA and ES. These clinicians are crucial in supporting their patients during treatment by ensuring that they have clear, concise information regarding available therapies and the correct application and maintenance of prescribed devices.

Prim Care Companion J Clin Psychiatry 2009;11(6):330-338

(c) Copyright 2009 Physicians Postgraduate Press, Inc.

Submitted: September 29, 2008; accepted November 25, 2008 (doi:10.4088/PCC.08m00725).

Corresponding author: Larry Culpepper, MD, MPH, Department of Family Medicine, Boston Medical Center, 771 Albany St, Dowling 5, Boston,MA02118 (Larry.Culpepper@bmc.org).
W hen sleep is disrupted in obstructive sleep apnea (OSA) by arousals, apneas (complete cessation of breathing), and hypopneas (partial obstruction of breathing), the consequences include cardiovascular morbidity, excessive sleepiness (ES), depressed mood, cognitive impairment, and diminished quality of life.

Obstructive sleep apnea and associated ES impose a significant societal and individual burden. For example, patients with OSA are at a significantly greater risk of having a traffic accident than individuals without OSA. ${ }^{1}$ More than 800,000 drivers were involved in OSA-related motor vehicle collisions in the United States during 2000 at a cost of 1,400 lives. ${ }^{2}$ Compared with a reference group from the general population, individuals with OSA are also at a higher risk of being involved in an occupational accident due to reduced wakefulness, vigilance, and attention. ${ }^{3}$ Furthermore, OSA and associated ES impair patients' quality of life, particularly vitality and social functioning. ${ }^{4}$ Psychiatric disorders are common in patients with OSA. In a study of a large cohort of nearly 120,000 veterans treated in US hospitals, anxiety, posttraumatic stress disorder, and psychosis were found in significantly more patients with OSA than in patients who did not have this sleep disorder $(16.7 \%, 11.9 \%$, and $5.1 \%$, respectively; $P<.0001){ }^{5}$

The aims of this review are to examine the relationship between OSA and depression and also to describe how OSA and ES can be recognized in the primary care setting. The role of the primary care physician (PCP) in supporting, monitoring, and educating patients during treatment for OSA will also be discussed.

\section{METHOD}

A MEDLINE search of articles published between 1990 and 2008 was conducted using the search terms $o b$ structive sleep apnea AND excessive sleepiness, obstructive sleep apnea AND depression, and obstructive sleep apnea AND primary care. Searches were limited to articles in English, clinical trials, meta-analyses, randomized controlled trials, and reviews. Search limits also excluded patients aged $\leq 19$ years. In total, 239 articles were identified; however, articles concerning other sleep disorders and forms of apnea were excluded, as were studies in which the focus was not primarily on OSA as a morbidity or comorbidity. The reference lists of articles thus 


\section{Clinical Points}

By being vigilant for risk factors, symptoms, and common comorbidities associated with OSA and ES, primary care physicians play a crucial role in recognizing this sleep disorder and facilitating appropriate patient referral, diagnosis, and treatment.

- A variety of treatments are available for patients with OSA and associated ES, including positive airway pressure devices, oral appliances, and surgery. The individual preferences and requirements of each patient will dictate the most appropriate treatment.

- Primary care physicians are instrumental in monitoring therapy and ensuring compliance in patients with OSA and associated ES; frequent follow-up is recommended to maximize patient comfort and understanding of his or her treatment modality.

identified were searched manually to find additional articles of interest. Publications cited include studies and reviews concerned with the prevalence of OSA and the diagnosis and management of its sequelae and associated comorbidities, with a particular focus on depression.

\section{DEPRESSION IN PATIENTS WITH OSA}

Depression is reported at a prevalence of $1.8 \%-3.3 \%$ in community-based studies of the general population. ${ }^{6}$ In patients with a sleep disorder, the prevalence of depression has been reported to be $17.6 \%^{7}$; in patients with OSA in particular, its prevalence is even higher, ranging from approximately $20 \%$ to $40 \%$ when assessed using the Beck Depression Inventory. ${ }^{5,8}$ Progression of OSA has been found to be associated with an increased risk of developing depression. ${ }^{10}$ Even patients with mild OSA are $60 \%$ more likely to become depressed than individuals without this sleep disorder. ${ }^{10}$

A robust causative relationship has been demonstrated between depression and fatigue in patients with OSA. ${ }^{11}$ Scores on the Center for Epidemiologic Studies Depression Scale were significantly correlated with scores on the Profile of Mood States Fatigue Scale in a study of 60 patients with OSA (correlation coefficient $=0.68, d f=58 ; P<.001) .{ }^{11}$ Furthermore, depression is a significant predictor of ES in obese patients with OSA (odds ratio $=1.69 ; 95 \%$ CI, 1.15-2.49; $P<$ $.01){ }^{12}$ It has been reported that the severity of neither OSA nor ES is correlated with the severity of fatigue in patients with this sleep disorder. These results indicate that depressive symptoms may be responsible for a proportion of the fatigue reported by patients with OSA. ${ }^{11}$ Both OSA and depression have profoundly detrimental effects on quality of life. ${ }^{13}$ Further evidence for a link between OSA and depression comes from the outcomes of treatment with continuous positive airway pressure (CPAP), which has been demonstrated to ameliorate this mood disorder in patients with OSA (as will be discussed later).

\section{PREVALENCE OF OSA AND ASSOCIATED ES}

In the United States, approximately $9 \%$ of women and $24 \%$ of men aged $\leq 60$ years are thought to have OSA. ${ }^{14}$ Mild OSA (Apnea-Hypopnea Index [AHI] > 5/h) occurs in approximately $20 \%$ of US adults with a mean body mass index of $25-28 \mathrm{~kg} / \mathrm{m}^{2}$ (defined as overweight), while approximately $7 \%$ of overweight adults have at least moderate OSA (AHI > 15/h) ${ }^{14-16}$ Furthermore, undiagnosed OSA with associated ES is estimated to be present in up to $5 \%$ of adults in Western countries. ${ }^{16}$ Increasing age and obesity are significant risk factors for OSA, and as these characteristics become more common, the prevalence of OSA is predicted to rise in the population. ${ }^{17} \mathrm{ES}$ is the most common symptom of OSA, but it can also occur as a result of insomnia, circadian rhythm sleep disorder, sleep-related movement disorders, primary hypersomnia, narcolepsy, insufficient sleep, drug use, and neurologic and psychiatric disorders. It is therefore often difficult to attribute ES to any one cause. ${ }^{17}$

\section{RECOGNITION AND DIAGNOSIS OF OSA AND ASSOCIATED ES}

\section{Recognition}

Recognition of OSA by PCPs requires assessment of the patient's history and physical and lifestyle risk factors. The PCP also needs to bear in mind that patients may not be aware that they have OSA or even that they are excessively sleepy. Frequent presenting symptoms of and risk factors for OSA are given in Table 1..$^{15,16,18-24}$

A number of illnesses in addition to depression are comorbid with OSA, including hypertension and diabetes. PCPs commonly see patients with these conditions and are therefore ideally placed to assess these high-risk patients for symptoms associated with OSA. ${ }^{25}$ For example, because depression and OSA are common comorbidities, maintaining a high level of clinical suspicion for OSA in patients with somatic symptoms of depression would 


\begin{tabular}{ll}
\hline \multicolumn{2}{l}{ Table 1. Frequent Presenting Symptoms and Risk Factors for } \\
Obstructive Sleep Apnea , $^{2,18-24}$ \\
\hline Presenting Symptoms & Risk Factors \\
\hline Excessive sleepiness & Male sex \\
Dry throat & Postmenopausal women \\
Snoring & Obesity or overweight \\
Choking during sleep & Large neck circumference \\
(including episodes & (women: $>16$ in and \\
reported by sleep partner) & men: $>17$ in) \\
Irritability & Craniofacial variation/ \\
Nocturia & pharyngeal crowding/ \\
Headache on waking & nasal obstruction \\
Resistant hypertension & Hypertension \\
and/or atrial fibrillation & Cerebrovascular disease \\
Decreased libido & Coronary artery disease \\
Unrefreshing sleep & Increasing age \\
& Smoking \\
& Alcohol use \\
& Sedative use \\
\hline
\end{tabular}

seem judicious. ${ }^{26}$ Furthermore, comprehensive assessment of comorbidities allows appropriate goal setting during treatment planning, provides a more accurate prognosis, and also facilitates suitable evaluation during follow-up visits. ${ }^{27}$

Patients with suspected OSA who have serious medical conditions or other sleep disorders or whose occupation or safety may be put at significant risk by OSA should be referred to a sleep specialist for consultation at the earliest opportunity. Rapid referral ensures that the diagnosis of OSA can be confirmed, its severity determined, and suitable treatment initiated in order to maintain the patient's well-being and avoid jeopardizing existing treatment regimens or exacerbating comorbidities. ${ }^{25}$

\section{Diagnosis}

In diagnosing OSA, it can be useful to exclude other causes of sleep-disordered breathing, ${ }^{28}$ although this is not always a simple process. For example, OSA and insomnia share symptoms; however, patients with insomnia require a longer time to fall asleep in sleep laboratory tests than those with ES associated with OSA, and a differential diagnosis can be made on this basis. Examination of the airway for patency and a measurement of neck circumference should also be carried out in patients with suspected OSA. Many clinicians advocate testing for thyroid-stimulating hormone in order to ascertain whether the symptoms of OSA could be due to an associated hypothyroid condition. ${ }^{29}$

Diagnosis of OSA in elderly patients can present a particular challenge for PCPs, as age-related changes can lead to individuals sleeping for shorter periods and experiencing tiredness and nocturia that are not directly attributable to OSA. In addition, older patients often receive multiple medications that cause restlessness or sleepiness as side effects; therefore, PCPs should check whether the patient's ES is an accompanying symptom of another condition or a side effect of a prescribed medication. ${ }^{27}$
A number of simple tests can be used in the clinic to aid the initial diagnosis of OSA. Use of oximetry (determination of the oxygen saturation of hemoglobin) by PCPs can be helpful in assessing and excluding patients in whom OSA is a possible but improbable diagnosis and who therefore may not warrant immediate referral to a sleep laboratory. ${ }^{25}$ However, oximetry does not replace full assessment of the patient in a sleep laboratory for confirmation of a diagnosis of OSA. ${ }^{25}$

Excessive sleepiness can be assessed using the Epworth Sleepiness Scale (ESS) ${ }^{30}$ in the primary care setting or by more complex testing in the sleep laboratory. The ESS is a simple questionnaire that requires patients to rate the likelihood that they will fall asleep in 8 different situations on a scale of 0 ("would never doze") to 3 ("high chance of dozing"). The situations rated range from those likely to induce sleep (eg, "watching TV") to more active states (eg, "sitting and talking with someone"). ${ }^{30}$ An ESS score $\geq 10$ is usually taken to be indicative of ES. Objective tests for ES used in the sleep laboratory include the Multiple Sleep Latency Test, which measures the time taken to fall asleep (using electroencephalograph criteria) in a darkened room, and the Maintenance of Wakefulness Test, in which the subject is instructed to stay awake rather than fall asleep. ${ }^{31,32}$

\section{Confirmation of Initial Diagnosis}

All patients with suspected OSA should be referred to a sleep specialist to determine whether polysomnography is required. Involvement of a sleep specialist at a relatively early stage of OSA diagnosis allows tailoring of the polysomnography to the individual needs of the patient and more accurate interpretation of results and also allows the PCP and sleep specialist to work together in planning, implementing, and following up on treatment. Polysomnography simultaneously records sleep and breathing patterns and is the standard diagnostic test used by specialists for sleep disorders. Polysomnography for OSA is sometimes conducted in an attended "split study," which takes place overnight at a sleep laboratory and consists of assessment and confirmation of the OSA diagnosis followed by CPAP titration. Recently, unattended portable polysomnography monitors that can be used at home have been approved for the diagnosis of OSA by the American Academy of Sleep Medicine (AASM), although only in patients with a high probability of positive diagnosis and only in conjunction with comprehensive sleep evaluation performed by a specialist. ${ }^{33}$ Medicaid and Medicare services require an assessment using either attended or portable polysomnography before authorization is given for CPAP treatment. 25,33

The occurrence of $\geq 5$ scoreable respiratory events per hour is used to define OSA. These events can include any combination of obstructive apneas, hypopneas, or arousals. There is currently no standard definition for the 


\begin{tabular}{|c|c|c|c|}
\hline \multirow[b]{2}{*}{ Characteristic } & \multicolumn{3}{|c|}{ Obstructive Sleep Apnea Severity } \\
\hline & Mild & Moderate & Severe \\
\hline Sleepiness & $\begin{array}{l}\text { Indicative ESS score }<12 \text {; sleepiness } \\
\text { present only when sedentary or } \\
\text { when little attention is required; } \\
\text { may not be present every day and } \\
\text { produces only minor impairment } \\
\text { of social and occupational functions }\end{array}$ & $\begin{array}{l}\text { Indicative ESS score } 13-17 \text {; sleepiness } \\
\text { occurs when minimally active or } \\
\text { when a moderate degree of attention } \\
\text { is required (eg, when driving or } \\
\text { watching a movie) }\end{array}$ & $\begin{array}{l}\text { Indicative ESS score 18-24; sleepiness } \\
\text { occurs during active tasks or tasks } \\
\text { that require significant attention (eg, } \\
\text { conversation, eating, or walking); } \\
\text { produces marked impairment of } \\
\text { social or occupational function }\end{array}$ \\
\hline $\begin{array}{l}\text { Gas exchange } \\
\text { abnormalities }\end{array}$ & $\begin{array}{l}\text { Mean oxygen saturation } \geq 90 \% \text { and } \\
\text { minimum oxygen saturation } \geq 85 \%\end{array}$ & $\begin{array}{l}\text { Mean oxygen saturation } \geq 90 \% \text { and } \\
\text { minimum oxygen saturation } \geq 70 \%\end{array}$ & $\begin{array}{l}\text { Mean oxygen saturation }<90 \% \text { or } \\
\text { minimum oxygen saturation }<70 \%\end{array}$ \\
\hline $\begin{array}{l}\text { Respiratory } \\
\text { disturbance }\end{array}$ & AHI: 5-15/h & AHI: $16-30 / h$ & AHI: > 30/h \\
\hline
\end{tabular}

${ }^{\mathrm{a} B a s e d}$ on Institute for Clinical Systems Improvement. ${ }^{25}$

${ }^{\mathrm{b}}$ The severity of obstructive sleep apnea and associated excessive sleepiness is determined by the most severe rating of the 3 domains. Abbreviations: AHI = Apnea-Hypopnea Index, ESS = Epworth Sleepiness Scale.

different levels of OSA severity; however, the Institute for Clinical Systems Improvement has given guideline recommendations for the determination of OSA severity (Table 2)..$^{25}$

\section{TREATMENT}

\section{Continuous Positive Airway Pressure}

Efficacy and tolerability of CPAP. CPAP is the gold standard of medical therapy for $\mathrm{OSA}^{34}$ and is recommended for the treatment of moderate to severe OSA and as an option for mild OSA (Figure 1). ${ }^{35-37}$ The recommendations of the AASM also recognize that CPAP can improve quality of life and is an effective adjunct to lower blood pressure in patients with hypertension and OSA. ${ }^{35}$ CPAP is adjusted to abolish apneas, hypopneas, and snoring and to diminish oxygen desaturation during all stages of sleep, thereby improving sleep quality. CPAP has also been shown to improve ES, depression, cognitive function, quality of life, blood pressure, and some cardiovascular variables, although further studies are required. . $^{23,38-42}$

Examples of theoretical disadvantages of CPAP are reduced renal function and cardiac output. Hemodynamic responses reported after treatment with CPAP are variable; CPAP has been reported to reduce cardiac output in healthy subjects in a number of studies, although cardiac output is increased by CPAP in patients with poorly compensated congestive heart failure. ${ }^{43,44}$ Conversely, it has also been reported that cardiac output may be reduced in some patients with severe congestive heart failure and that no effect is evident with CPAP in control subjects. ${ }^{45,46}$ Renal blood flow and function have also been reported to decrease with increasing CPAP air pressure. ${ }^{47}$ Until further information is available concerning the effects of $\mathrm{CPAP}$ on renal and cardiac function, it is difficult to make recommendations; however, CPAP is currently the most effective treatment for OSA, and the issues highlighted by this small number of studies are likely to be applicable only in patients with significant comorbidities. Nose bleeds, dermatitis, and nasal bridge sores may also occur during CPAP use but are usually associated with incorrect pressure/use or inadequate fit of the device and can be obviated by carefully fitting and adjusting the device at treatment onset and monitoring the patient throughout its use.

Varieties of positive airway pressure devices. Variations of CPAP therapy include bilevel positive airway pressure (BiPAP) and automatic self-adjusting positive airway pressure (APAP) devices (Figure 1). By applying lower pressure during expiration, BiPAP mimics normal breathing more closely than CPAP. BiPAP is therefore a form of assisted ventilation that may be better tolerated by some patients with OSA and particularly by those with chronic obstructive pulmonary disease, hyperventilation syndrome, or awake hypercapnia. ${ }^{25}$ APAP devices vary the pressure of air delivered to the patient, providing the lowest pressure possible to keep the airway patent. Again, this device may be suitable for patients who find it difficult to tolerate constant pressure when using $\mathrm{CPAP}^{25}$

CPAP in the treatment of depression associated with OSA. Treatment with CPAP has been shown to significantly decrease depressive symptoms in patients with OSA when assessed using the Beck Depression Inventory at baseline, after 4-6 weeks' treatment, and at 1 year $(P<.05) .^{9}$ Earlier, short-term studies support this finding. ${ }^{48-51}$ In addition, a recent case study has reported that suicidal ideation and depression were rapidly resolved in a patient with severe OSA after initiation of CPAP treatment. ${ }^{52}$ Conversely, a number of studies have reported that CPAP does not affect depression scores in patients with $\mathrm{OSA}^{53-56}$; however, a comprehensive meta-analysis has revealed that all of these negative studies investigated patients who had mild depression at baseline or excluded patients with major depressive disorder. ${ }^{26}$ This study design may have skewed the study outcomes toward a negative result, as it has been reported previously that patients with OSA and more severe mood disorders receive the greatest benefit from CPAP. ${ }^{57}$ 
Figure 1. Positive Airway Pressure Devices for Treatment of Obstructive Sleep Apnea

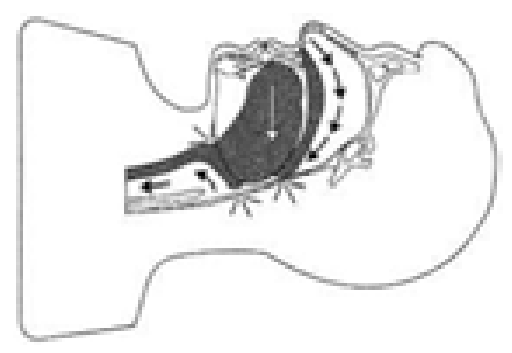

Partially blocked upper airway increases inspiratory effort and the vibrations of soft tissue that cause snoring

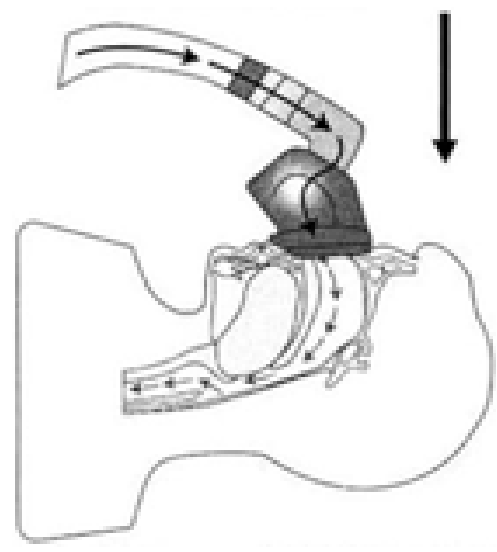

Although the tongue and soft tissue have fallen to the back of the throat, the pressure of the air pumped in by the PAP device holds the upper airway open
- Positive airway pressure (PAP) devices deliver pressurized air via a close-fitting nasal or oronasal mask to support the pharyngeal airway and prevent the soft tissue from collapsing

- PAP devices provide continous positive airway pressure (CPAP); CPAP is the gold standard

- Approximately $20 \%$ of patients with obstructive sleep apnea find it difficult to exhale against the constant airway pressure that is a feature of $\mathrm{CPAP}^{36,37}$

- With bilevel positive airway pressure (BiPAP), air delivered through the mask can be set at one pressure for inhaling and another for exhaling

- Automatic self-adjusting positive airway pressure devices measure the amount of airway obstruction and adjust to provide the necessary amount of positive airway pressure

- Patients' use of PAP devices should be monitored and support given when necessary to promote persistence with the therapy
Improvement in the symptoms of depression after treatment of OSA with CPAP indicates that there is a causal association between these illnesses. The mechanism that underlies this relationship has yet to be clearly delineated, although the effects on mood of sleep fragmentation and intermittent hypoxia may play a substantive role in the etiology of depression in patients with OSA. ${ }^{8,10}$ Indeed, 1 study has reported that the severity of psychological symptoms associated with OSA was negatively related to total sleep time and the percentage of stage 2 nonrapid eye movement sleep. ${ }^{58}$

In addition to CPAP therapy, it is important to prescribe suitable treatment for depression in patients with OSA who have this comorbidity to reduce both the mood disturbance and associated fatigue. However, depressive symptoms may be not only a comorbidity associated with OSA but also a consequence of the sleep disorder itself, and treatment with antidepressants or counseling will not be effective in patients with OSA who have been misdiagnosed with depression.
Monitoring CPAP treatment. Follow-up of patients receiving treatment for OSA is essential and is optimally performed in the primary care setting. In the initial stages of treatment, follow-ups should include assessment of adherence and acceptability of CPAP, adequacy of education regarding the device, and involvement in patient support groups. It is also vital to discuss any potential problems, such as nasal dryness, pain, and issues with the CPAP device supplier. Persistence or resolution of symptoms, such as snoring, should also be checked, taking into account the perceptions of the patient's sleep partner as well as subjective improvements in daytime activities and quality of life. A key follow-up measure for patients being treated with CPAP is ES, which is usually assessed using the ESS. If residual ES is detected, treatment with adjunctive pharmaceutical agents may be appropriate and useful.

Use of CPAP (hours per night) during the first few weeks has been found to predict long-term compliance, and patients using CPAP for $<2$ hours per night are more likely to withdraw from treatment. ${ }^{59}$ Furthermore, 


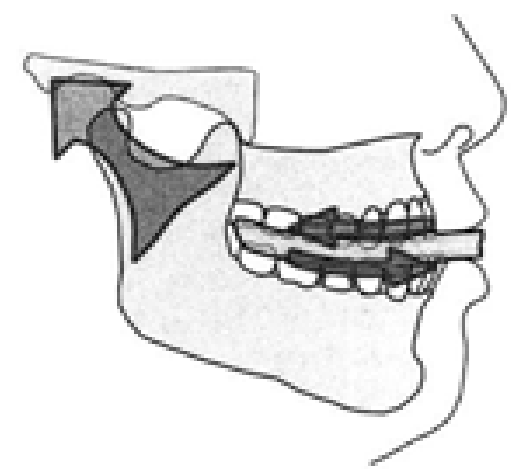

- The most effective oral appliance is the mandibular advancement device, which is fitted to the maxillary and mandibular dentition and moves the position of the lower jaw forward increasing pharyngeal size and advancing the base of the tongue, thus reducing airway instability

- Current evidence suggests that oral devices can improve apnea and daytime sleepiness and may improve blood pressure ${ }^{66}$

- Some patients experience excessive salivation, temporomandibular joint discomfort, and dental adverse events with oral devices ${ }^{22,67}$

- Long-term adverse events are unclear²2,67

${ }^{a}$ Adapted with permission from George. ${ }^{65}$

a dose-response relationship has been demonstrated between nightly hours of CPAP use and improvements in sleepiness and daily functioning. ${ }^{60}$ Patients whose ES was resolved during treatment (ie, their ESS score reduced to < 10) used CPAP for approximately 1 hour more each night than study participants who reported experiencing residual ES (5.1 versus 4.0 hours of CPAP use per night, respectively). ${ }^{60}$ Patients with higher depression scores have been reported to have lower compliance with CPAP, ${ }^{61}$ and the interventions discussed below may be of particular use in this patient population as an aid to compliance.

If the patient has persistent difficulty with exhalation or dry mouth, alternative positive airway pressure devices may be more suitable (Figure 1). Gradual desensitization to the CPAP mask may also improve compliance. Desensitization involves the patient wearing the mask for short periods during the day while in an upright position and using low air pressures before raising the pressure to the required level. ${ }^{62}$ White noise machines may also be helpful in maintaining compliance in patients who find that the noise of a CPAP machine disrupts their sleep, while heated humidification of CPAP air may improve comfort in patients with nasal congestion and dryness. $^{25,63}$

Patients should be made aware of appropriate CPAP device maintenance to ensure that they receive optimum treatment and to increase the lifespan of their machine. The CPAP mask should be wiped daily and, along with the humidifier, should be washed thoroughly each week. Maintenance and support vary between machines and providers. The CPAP provider should supply new head gear and filters when required and should also alter the machine to use the correct air pressure when prescribed by the physician; these goods and services should be covered by the patient's medical insurance. ${ }^{64}$
Long-term follow-up of patients treated with CPAP should include an annual discussion regarding adherence, comfort, and fit; consideration of any requirement for additional education on maintenance of the equipment; and patient reassessment if their weight has increased or decreased by $>10 \%$ or if new comorbidities have developed. Patients with OSA may raise queries about CPAP use if they have the flu or upper respiratory tract infections. It is important to reassure them that they should maintain compliance with their usual CPAP regimen and use humidification, decongestants, nasal sprays, and nasal saline if they find them helpful. ${ }^{25}$

\section{Oral Appliances}

Oral appliances are an alternative treatment approach for patients with OSA who are unable or unwilling to use CPAP (Figure 2). ${ }^{65}$ A recent Cochrane review reported that oral appliances are not as effective as CPAP at improving sleep-disordered breathing. ${ }^{22,66,67}$ Patients may prefer oral appliances to CPAP because of their ease of use, and they may therefore yield equivalent health outcomes in some patients due to better patient adherence. ${ }^{68}$ If a patient stipulates a preference for an oral appliance or oral surgery for his or her OSA, it may be useful to consult the American Academy of Dental Sleep Medicine Web site (www.dentalsleepmed.org) to find additional information and details of appropriate specialists.

\section{Surgery}

Overall, surgical intervention (apart from tracheostomy) is not as consistently effective as CPAP, nor can its efficacy be predicted according to patient phenotype. ${ }^{69}$ Tonsillectomy/adenoidectomy is, however, indicated for the treatment of OSA in children. ${ }^{70}$ Surgical procedures for OSA in patients with identifiable upper airway or craniofacial abnormalities include removal of 
large tonsils and resection of the uvula and soft palate to enlarge the oropharynx. ${ }^{71}$ Other surgical options include craniofacial reconstruction and tracheostomy (this corrects the condition as it bypasses the obstruction). ${ }^{71} \mathrm{An}$ indepth discussion of surgical interventions for the treatment of OSA is beyond the scope of this article, but there are a number of comprehensive reviews that cover this topic. ${ }^{72,73}$ Foremost among these articles is a Cochrane review that concludes that the available studies do not support the use of surgery for patients with OSA due to a lack of consistent demonstrable benefit. ${ }^{74}$

\section{Other Therapies}

Common forms of self-help recommended as adjunctive treatments to primary therapy with CPAP include weight loss (if applicable, including bariatric surgery in the morbidly obese), avoidance of alcohol and sedatives, sleeping in a more upright position, and the use of nasal dilation clips. ${ }^{25,75,76}$ In addition, supplemental oxygen may be used as an adjunct to other therapies for OSA; however, it does not address morbidities associated with this sleep disorder in the same way as CPAP treatment, and it has not yet been rigorously studied in randomized trials. ${ }^{77}$ For these reasons, supplemental oxygen is not indicated as a primary treatment for OSA. ${ }^{76}$

\section{EVALUATION AND TREATMENT OF RESIDUAL ES}

Residual ES after treatment of OSA with CPAP is a commonly encountered problem for the patient and his or her PCP and can be identified using the ESS. Even with optimal CPAP therapy, up to half of patients with OSA have residual ES. ${ }^{60}$ In patients compliant with CPAP therapy but who still experience ES, adjunctive pharmacologic therapy is a treatment option. Before introducing such therapy, it is important to ensure that CPAP is being used appropriately by checking the air pressure of the device and the level of patient compliance. Other potential causative factors, such as another sleep disorder, should also be reassessed at this stage.

Modafinil has been approved by the US Food and Drug Administration and is recommended by the AASM for the management of residual ES in patients with OSA as adjunctive therapy to nasal CPAP. ${ }^{76,77}$ In addition, armodafinil, the R-enantiomer of modafinil (which is a racemic mixture of the $\mathrm{R}$ - and $\mathrm{S}$-enantiomers) has been evaluated in patients with ES associated with OSA and has shown statistically significant improvements in the maintenance of wakefulness versus placebo. ${ }^{78-80}$ Modafinil and armodafinil are generally well tolerated in patients with OSA. ${ }^{81-84}$ It is important to note, however, that neither modafinil nor armodafinil addresses the underlying obstruction that causes OSA and must be used in conjunction with CPAP to avoid the return of symptoms and cardiovascular complications. Patients should be counseled to ensure compliance with CPAP when using adjunctive pharmacotherapy.

\section{DISCUSSION}

OSA is prevalent in patients presenting in the primary care setting and will become more common as the global population becomes more overweight and ages. This is particularly disquieting when it is considered that both OSA and associated ES have far-reaching consequences for the individual, in terms of morbidity, mortality, and reduced quality of life, as well as for public health, due to the economic burden and the impact that these disorders have on traffic- and occupation-related accidents. Only through appropriate treatment can the morbidity and mortality associated with OSA be alleviated. Depression is common in patients with OSA and is both a comorbidity and a consequence of this sleep disorder.

CPAP is the mainstay of therapy for OSA, and early recognition and treatment of OSA with CPAP may improve comorbid conditions, including depression. Despite CPAP's efficacy in treating OSA, ES may persist in some patients. Modafinil and armodafinil are effective adjunctive therapies to improve wakefulness in these patients.

Primary care physicians play a key role in recognizing the characteristic symptoms of OSA and being vigilant for patients not reporting symptoms but with risk factors and comorbidities suggestive of OSA, such as depression. Conversely, PCPs should also look for and address depression in patients with OSA. Furthermore, treatment of OSA is enhanced if PCPs work closely with sleep specialists to optimize patient adherence and response to therapy. A critical role for the PCP is in monitoring the progress of the patient and addressing treatment issues that may arise.

\begin{abstract}
Drug names: armodafinil (Nuvigil), modafinil (Provigil).
Author affiliations: Department of Family Medicine, Boston Medical Center, Massachusetts (Dr Culpepper) and the Sleep Disorders and Research Center, Henry Ford Hospital, Detroit, Michigan (Dr Roth). Potential conflicts of interest: Dr Culpepper has served as a consultant to AstraZeneca, Cephalon, Eli Lilly, Forest, Pfizer, Takeda, and Wyeth and on the speakers' or advisory boards of Forest and Wyeth. Dr Roth has served as a consultant in the past 12 months to Abbott, Actelion, Arena, BTG, Evotec, Intec, Intracellular, Jazz, Merck, Neurim, Neurocrine, Neurogen, Novartis, Organon, Proctor \& Gamble, Pfizer, sanofi-aventis, Schering-Plough, Sepracor, Shire, Somaxon, Takeda, TransOral, and Vanda.

Funding/support: Editorial support was provided by Jane Bryant, $\mathrm{PhD}$, of Anthemis Consulting Ltd, Cheshire, United Kingdom, and was funded by Cephalon Inc, Frazer, Pennsylvania, who provided a medical accuracy review. The authors were not compensated and retained full editorial control over the content of the article.
\end{abstract}

\section{REFERENCES}

1. Terán-Santos J, Jiménez-Gómez A, Cordero-Guevera J. The association between sleep apnea and the risk of traffic accidents. $N$ Engl J Med. 1999;340(11):847-851.

2. Sassani A, Findley LJ, Kryger M, et al. Reducing motor-vehicle 
collisions, costs and fatalities by treating obstructive sleep apnea syndrome. Sleep. 2004;27(3)453-458.

3. Ulfberg J, Carter N, Edling C. Sleep-disordered breathing and occupational accidents. Scand J Work Environ Health. 2000;26(3):237-242.

4. Sforza E, De Saint Hilaire Z, Pelissolo A, et al. Personality, anxiety and mood traits in patients with sleep-related breathing disorders: effect of reduced daytime alertness. Sleep Med. 2002;3(2):139-145.

5. Sharafkhaneh A, Giray N, Richardson P, et al. Association of psychiatric disorders and sleep apnea in a large cohort. Sleep. 2005;28(11): $1405-1411$.

6. Pignone MP, Gaynes BN, Rushton JL, et al. Screening for depression in adults: a summary of the evidence for the US Preventive Service Task Force. Ann Intern Med. 2002;136(10):765-776.

7. Ohayon MM. The effects of breathing-related sleep disorders on mood disturbances in the general population. J Clin Psychiatry. 2003;64(10): 1195-1200.

8. Hashmi AM, Giray N, Hirshkowitz M. Sleep-related breathing disorders and mood disorders. Sleep Med Clin. 2006;1(4):513-517.

9. Schwartz DJ, Karatinos G. For individuals with obstructive sleep apnea, institution of CPAP therapy is associated with an amelioration of symptoms of depression which is sustained long term. J Clin Sleep Med. 2007; 3(6):631-635.

10. Peppard PE, Szklo-Coxe M, Hla KM, et al. Longitudinal association of sleep-related breathing disorder and depression. Arch Intern Med. 2006; 166(16):1709-1715

11. Bardwell WA, Moore P, Ancoli-Israel S, et al. Fatigue in obstructive sleep apnea: driven by depressive symptoms instead of apnea severity? Am J Psychiatry. 2003;160(2):350-355.

12. Basta M, Lin HM, Pejovic S, et al. Lack of regular exercise, depression, and degree of apnea are predictors of excessive daytime sleepiness in patients with sleep apnea: sex differences. J Clin Sleep Med. 2008;4(1): $19-25$.

13. Akashiba T, Kawahara S, Akahoshi T, et al. Relationship between quality of life and mood or depression in patients with severe obstructive sleep apnea syndrome. Chest. 2002;122(3):861-865.

14. Young T, Palta M, Dempsey J, et al. The occurrence of sleep-disordered breathing among middle-aged adults. $N$ Engl J Med. 1993;328(17): 1230-1235.

15. Bixler EO, Vgontzas AN, Ten Have T, et al. Effects of age on sleep apnea in men, 1: prevalence and severity. Am J Respir Crit Care Med. 1998; 157(1):144-148.

16. Young T, Peppard PE, Gottlieb DJ. Epidemiology of obstructive sleep apnea: a population health perspective. Am J Respir Crit Care Med. 2002;165(9):1217-1239.

17. Pagel JF. The burden of obstructive sleep apnea and associated excessive sleepiness. J Fam Pract. 2008;57(suppl 8):S3-S8.

18. Dancey DR, Hanly PJ, Soong C, et al. Gender differences in sleep apnea: the role of neck circumference. Chest. 2003;123(5):1544-1550.

19. Davies RJ, Stradling JR. The relationship between neck circumference, radiographic pharyngeal anatomy, and the obstructive sleep apnea syndrome. Eur Respir J. 1990;3(5):509-514.

20. Flemons WW. Clinical practice: obstructive sleep apnea. $N$ Engl J Med. 2002;347(7):498-504.

21. Peppard PE, Young T, Palta M, et al. Longitudinal study of moderate weight change and sleep-disordered breathing. JAMA. 2000;284(23): 3015-3021.

22. Sanders MH, Givelber RJ. Overview of obstructive sleep apnoea in adults. In: Lee-Chong, ed. Sleep Disorders: A Brief Guide. Chichester, UK: John Wiley and Sons; 2007:39-57

23. Scottish Intercollegiate Guidelines Network. Management of Obstructive Sleep Apnoea/Hypopnoea Syndrome in Adults: A National Clinical Guideline. Edinburgh, UK: Scottish Intercollegiate Guidelines Network; 2003:73.

24. Young T, Shahar E, Nieto FJ, et al. Predictors of sleep-disordered breathing in community-dwelling adults: the Sleep Heart Health Study. Arch Intern Med. 2002;162(8):893-900.

25. Institute for Clinical Systems Improvement. Health Care Guideline; Diagnosis and Treatment of Obstructive Sleep Apnea; 2008. http://www.icsi.org/sleep_apnea/ sleep_apnea_diagnosis_and_treatment_of_obstructive_html. Accessed November 2, 2009

26. Schröder CM, O'Hara R. Depression and obstructive sleep apnea (OSA). Ann Gen Psychiatry. 2005;4:13.
27. Doghramji PP. Recognition of obstructive sleep apnea and associated excessive sleepiness in primary care. J Fam Pract. 2008;57(suppl 8):S17-S23.

28. Rakel RE. Recognition and diagnosis of excessive daytime sleepiness from a primary care perspective. Consultant. 2007;47(suppl 10):22-30.

29. Skjodt NM, Atkar R, Easton PA. Screening for hypothyroidism in sleep apnea. Am J Respir Crit Care Med. 1999;160(2):732-735.

30. Johns MW. Daytime sleepiness, snoring, and obstructive sleep apnea. Chest. 1993;103(1):30-36.

31. Carskadon MA, Dement WC, Mitler MM, et al. Guidelines for the Multiple Sleep Latency Test (MSLT): a standard measure of sleepiness. Sleep. 1986;9(4):519-524.

32. Mitler MM, Gujavarty KS, Browman CP. Maintenance of Wakefulness Test: a polysomnographic technique for evaluating treatment efficacy in patients with excessive somnolence. Electroencephalogr Clin Neurophysiol. 1982;53(6):658-661.

33. Collop NA, Anderson WM, Boehlecke B, et al. Clinical guidelines for the use of unattended portable monitors in the diagnosis of obstructive sleep apnea in adult patients. J Clin Sleep Med. 2007;3(7)737-747.

34. Sullivan CE, Issa FG, Berthon-Jones M, et al. Reversal of obstructive sleep apnoea by continuous positive airway pressure applied through the nares. Lancet. 1981;1(8225):862-865.

35. Kushida CA, Littner MR, Hirshkowitz M, et al. Practice parameters for the use of continuous and bilevel positive airway pressure devices to treat adult patients with sleep-related breathing disorders. Sleep. 2006; 29(3):375-380.

36. Meurice JC, Dore P, Paquereau J, et al. Predictive factors of long-term compliance with nasal continuous positive airway pressure treatment in sleep apnea syndrome. Chest. 1994;105(2):429-433.

37. Pieters T, Collard P, Aubert G, et al. Acceptance and long-term compliance with nCPAP in patients with obstructive sleep apnoea syndrome. Eur Respir J. 1996;9(5):939-944.

38. Bazzano LA, Khan Z, Reynolds K, et al. Effect of nocturnal nasal continuous positive airway pressure on blood pressure in obstructive sleep apnea. Hypertension. 2007;50(2)417-423.

39. Egea CJ, Aizpuru F, Pinto JA, et al. Cardiac function after CPAP therapy in patients with chronic heart failure and sleep apnea: a multicenter study. Sleep Med. 2008;9(6):660-666.

40. Gay P, Weaver T, Loube D, et al. Evaluation of positive airway pressure treatment for sleep related breathing disorders in adults. Sleep. 2006; 29(3)381-401.

41. Giles TL, Lasserson TJ, Smith BH, et al. Continuous positive airways pressure for obstructive sleep apnoea in adults. Cochrane Database Syst Rev. 2006;(3):CD001106.

42. Patel SR, White DP, Malhotra A, et al. Continuous positive airway pressure therapy for treating sleepiness in a diverse population with obstructive sleep apnea: results of a meta-analysis. Arch Intern Med. 2003;163(5):565-571.

43. de Hoyos A, Liu PP, Bernard DC, et al. Haemodynamic effects of continuous positive airway pressure in humans with normal and impaired left ventricular function. Clin Sci (Lond). 1995;88(2):173-178.

44. Montner PK, Greene ER, Murata GH, et al. Hemodynamic effects of nasal and face mask continuous positive airway pressure. Am J Respir Crit Care Med. 1994;149(6):1614-1618

45. Leech JA, Ascah KJ. Haemodynamic effects of nasal CPAP examined by Doppler echocardiography. Chest. 1991;99(2):323-326.

46. Liston R, Deegan PC, McCreery C, et al. Haemodynamic effects of nasal continuous positive airway pressure in severe congestive heart failure. Eur Respir J. 1995;8(3):430-435.

47. Sharkey RA, Mulloy EM, Long M, et al. The effect of continuous positive airway pressure (CPAP) on renal vascular resistance: the influence of renal denervation. Crit Care. 1999;3(1):33-37.

48. Engleman HM, Cheshire KE, Deary IJ, et al. Daytime sleepiness, cognitive performance and mood after continuous positive airway pressure for the sleep apnoea/hypopnoea syndrome. Thorax. 1993;48(9): 911-914.

49. Engleman HM, Martin SE, Deary IJ, et al. Effect of CPAP therapy on daytime function in patients with mild sleep apnoea/hypopnoea syndrome. Thorax. 1997;52(2):114-119.

50. Means MK, Lichstein KL, Edinger JD, et al. Changes in depressive symptoms after continuous positive airway pressure treatment for obstructive sleep apnea. Sleep Breath. 2003;7(1):31-42.

51. Sánchez AI, Buela-Casal G, Bermúdez MP, et al. The effects of 
continuous positive air pressure treatment on anxiety and depression levels in apnea patients. Psychiatry Clin Neurosci. 2001;55(6):641-646.

52. Krahn LE, Miller BW, Bergstrom LR. Rapid resolution of intense suicidal ideation after treatment of severe obstructive sleep apnea. J Clin Sleep Med. 2008;4(1):64-65.

53. Borak J, Cieślicki JK, Koziej M, et al. Effects of CPAP treatment on psychological status in patients with severe obstructive sleep apnoea. J Sleep Res. 1996;5(2):123-127.

54. Henke KG, Grady JJ, Kuna ST. Effect of nasal continuous positive airway pressure on neuropsychological function in sleep apnea-hypopnea syndrome: a randomized, placebo-controlled trial. Am J Respir Crit Care Med. 2001;163(4):911-917.

55. Muñoz A, Mayoralas LR, Barbé F, et al. Long-term effects of CPAP on daytime functioning in patients with sleep apnoea syndrome. Eur Respir J. 2000;15(4):676-681.

56. Yu BH, Ancoli-Israel S, Dimsdale JE. Effect of CPAP treatment on mood states in patients with sleep apnea. J Psychiatr Res. 1999;33(5):427-432.

57. Millman RP, Fogel BS, McNamara ME, et al. Depression as a manifestation of obstructive sleep apnea: reversal with nasal continuous positive airway pressure. J Clin Psychiatry. 1989;50(9):348-351.

58. Yue W, Hao W, Liu P, et al. A case-control study on psychological symptoms in sleep apnea-hypopnea syndrome. Can J Psychiatry. 2003; 48(5)318-323.

59. McArdle N, Devereux G, Heidarnejad H, et al. Long-term use of CPAP therapy for sleep apnea/hypopnea syndrome. Am J Respir Crit Care Med. 1999;159(4, pt 1):1108-1114.

60. Weaver TE, Maislin G, Dinges DF, et al. Relationship between hours of CPAP use and achieving normal levels of sleepiness and daily functioning. Sleep. 2007;30(6):711-719.

61. Edinger JD, Carwile S, Miller P, et al. Psychological status, syndromatic measures, and compliance with nasal CPAP therapy for sleep apnea. Percept Mot Skills. 1994;78(3, pt 2):1116-1118.

62. American Sleep Apnea Association. If Your Patient Is Not Complying With CPAP; 2007. http://www.sleepapnea.org/info/practitioner/ cpappatient.html.

63. Neill AM, Wai HS, Bannan SP, et al. Humidified nasal continuous positive airway pressure in obstructive sleep apnoea. Eur Respir J. 2003; 22(2):258-262.

64. American Sleep Apnea Association. Education Bulletin: Choosing a CPAP; 2005. http://www.sleepapnea.org/resources/pubs/cpap.htm.

65. George PT. Selecting sleep-disordered breathing appliances: biomechanical considerations. J Am Dent Assoc. 2001;132(3):339-347.

66. Lim J, Lasserson TJ, Fleetham J, et al. Oral appliances for obstructive sleep apnoea. Cochrane Database Syst Rev. 2006;(1):CD004435.

67. Gotsopoulos H, Chen C, Qian J, et al. Oral appliance therapy improves symptoms in obstructive sleep apnea: a randomized controlled trial. Am J Respir Crit Care Med. 2002;166(5):743-748.

68. Chan AS, Lee RW, Cistulli PA. Dental appliance treatment for obstructive sleep apnea. Chest. 2007;132(2):693-699.
69. Sher AE, Schechtman KB, Piccirillo JF. The efficacy of surgical modifications of the upper airway in adults with obstructive sleep apnea syndrome. Sleep. 1996;19(2):156-177.

70. Mitchell RB, Kelly J. Outcomes and quality of life following adenotonsillectomy for sleep-disordered breathing in children. ORL J Otorhinolaryngol Relat Spec. 2007;69(6):345-348.

71. Ballard RD. Management of patients with obstructive sleep apnea. J Fam Pract. 2008;57(suppl 8):S24-S30.

72. Li KK. Surgical therapy for obstructive sleep apnea syndrome. Semin Respir Crit Care Med. 2005;26:80-88.

73. Senders CW, Strong EB. The surgical treatment of obstructive sleep apnea. Clin Rev Allergy Immunol. 2003;25:213-220.

74. Sundaram S, Bridgman SA, Lim J, et al. Surgery for obstructive sleep apnoea. Cochrane Database Syst Rev. 2005;(4):CD001004.

75. Höijer U, Ejnell H, Hedner J, et al. The effects of nasal dilation on snoring and obstructive sleep apnea. Arch Otolaryngol Head Neck Surg. 1992;118(3):281-284.

76. Morgenthaler TI, Kapen S, Lee-Chiong T, et al. Practice parameters for the medical therapy of obstructive sleep apnea. Sleep. 2006;29(8): 1031-1035.

77. Veasey SC, Guilleminault C, Strohl KP, et al. Medical therapy for obstructive sleep apnea: a review by the Medical Therapy for Obstructive Sleep Apnea Task Force of the Standards of Practice Committee of the American Academy of Sleep Medicine. Sleep. 2006;29(8):1036-1044.

78. Black JE, Hirshkowitz M. Modafinil for treatment of residual excessive sleepiness in nasal continuous positive airway pressure treated obstructive sleep apnea/hypopnea syndrome. Sleep. 2005;28(4):464-471.

79. Hirshkowitz M, Black JE, Wesnes K, et al. Adjunct armodafinil improves wakefulness and memory in obstructive sleep apnea/hypopnea syndrome. Respir Med. 2007;101(3):616-627.

80. Roth T, White D, Schmidt-Nowara W, et al. Effects of armodafinil in the treatment of residual excessive sleepiness associated with obstructive sleep apnea/hypopnea syndrome: a 12-week, multicenter, double-blind, randomized, placebo-controlled study in nCPAP-adherent adults. Clin Ther. 2006;28(5):689-706.

81. Kingshott RN, Vennelle M, Coleman EL, et al. Randomized, doubleblind, placebo-controlled crossover trial of modafinil in the treatment of residual excessive daytime sleepiness in the sleep apnea/hypopnea syndrome. Am J Respir Crit Care Med. 2001;163(4):918-923.

82. Pack AI, Black JE, Schwartz JR, et al. Modafinil as adjunct therapy for daytime sleepiness in obstructive sleep apnea. Am J Respir Crit Care Med. 2001;164(9):1675-1681.

83. Schwartz JR, Hirshkowitz M, Erman MK, et al. Modafinil as adjunct therapy for daytime sleepiness in obstructive sleep apnea: a 12-week, open-label study. Chest. 2003;124(6):2192-2199.

84. Roth T, Rippon GA, Arora S. Armodafinil improves wakefulness and long-term episodic memory in nCPAP-adherent patients with excessive sleepiness associated with obstructive sleep apnea. Sleep Breath. 2008; 12(1):53-62. 